\title{
PDIA4 Correlates with Poor Prognosis and is a Potential Biomarker in Glioma
}

This article was published in the following Dove Press journal:

OncoTargets and Therapy

Haoyu $\mathrm{Li}^{\prime}$
Qing Liu'
Kai Xiao'
Zhengxi He
Chao $\mathrm{Wu}^{3}$
Jianjun Sun
Xin Chen
Suhua $\mathrm{Chen}^{3}$
Jun Yang
Qianquan $\mathrm{Ma}^{3}$
Jun Su

'Department of Neurosurgery, Xiangya Hospital, Central South University, Changsha 4I0008, Hunan, People's Republic of China; ${ }^{2}$ Department of Oncology, Xiangya Hospital, Central South University, Changsha 410008, Hunan, People's Republic of China; ${ }^{3}$ Department of Neurosurgery, Peking University Third Hospital, Peking University, Beijing 100191, People's Republic of China; ${ }^{4}$ Department of Neurosurgery, Hunan Children's Hospital, Changsha 410007, Hunan, People's Republic of China
Correspondence: Qianquan Ma; Jun Su Emailmqq0716@live.com; neurodoc_su@।26.com

\begin{abstract}
Purpose: Gliomas, characterized by aggressiveness and invasiveness, remain incurable after conventional therapies. The molecular mechanisms driving the progression and maintenance of glioma are still poorly understood.

Methods: The TCGA and CGGA databases were chosen for bioinformatics analysis. Gene expression profiling interactive analysis (GEPIA) was performed for differential analysis. The Kaplan-Meier method was chosen for survival analysis. Analysis of stromal and immune infiltration was performed using the ESTIMATE algorithm and xCell package. qPCR and Western blotting were performed to measure the expression of PDIA4 at the mRNA and protein levels. IHC was performed to detect the expression of PDIA4 in glioma tissues. The viability of glioma cells was evaluated by the CCK8 assay.

Results: In this study, we identified high PDIA4 expression in gliomas that correlated with poor prognosis. The association between IDH1 and different glioma patterns also indicated the potential biological role of PDIA4 in tumor development. Mechanistically, PDIA4 interacted with multiple immunological components to promote an immunosuppressive tumor microenvironment (TME). Knockdown of PDIA4 significantly impaired the proliferation of GBM cells.
\end{abstract}

Conclusion: Our results confirm that PDIA4 is an efficient biomarker of gliomas, with clinical implications for prognosis and therapeutic strategies.

Keywords: PDIA4, glioma, prognosis, biomarker, immune cells

\section{Introduction}

Gliomas are one of the most common malignant tumors in the central nervous system (CNS) and account for nearly $75 \%$ of primary tumors in adults. ${ }^{1}$ According to the histopathological features and prognostic factors, the World Health Organization (WHO) classifies gliomas into four grades (I-IV), from which the glioblastomas (GBMs) are categorized as the most malignant subtype (grade IV). ${ }^{2}$ Traditional multimodal therapeutic strategies against gliomas, including advanced neurosurgery, radiotherapy and chemotherapy, are unable to improve the prognosis of glioma patients. GBM patients have a poorer prognosis, with a median overall survival time of less than 17 months. ${ }^{3}$ Tolerance against multiple treatments and the invariable relapse of gliomas have been extensively studied and known to be caused by certain molecular or chromosomal subtypes, oncogenic activations, and the distinct metabolic immunosuppressive tumor microenvironment (TME). ${ }^{3-6}$ Pursuing a better understanding of the molecular landscape in gliomas, clinically significant novel markers have been detected. Various discoveries, such as promoter mutations in TERT, mutations in IDH1/IDH2, co-deletion of chromosome arms 1p/ 
19q, and H2K27M-mutant are clearly associated with improved homogeneity in clinical outcomes and are referred to as critical predictors in clinical practice. ${ }^{3,7-9}$ Further strengthening the knowledge of such molecular alterations will improve our perception of gliomas. In this regard, investigating novel molecular biomarkers or driver genes will facilitate the understanding of tumor promotion and the development of better therapeutic strategies to cure this disease.

Protein disulfide isomerases (PDIs) were originally discovered in the endoplasmic reticulum (ER) and were known to participate in protein folding. ${ }^{10}$ Encoded by the $\mathrm{P} 4 \mathrm{HB}$ gene, $\mathrm{PDI}$ is a $57-\mathrm{kDa}$ redox-dependent protein with a multi-domain structure. ${ }^{11}$ As critical ER enzymes, PDIs are primarily involved in oxidoreductase and chaperone activities that mediate the redox state in the cell and maintain proper protein folding and function. ${ }^{11,12}$ The biological functions of PDIs as reductases, oxidases, and chaperones in the ER have been associated with abundant physiopathological mechanisms, such as infection, coagulation, cellular viability, neurodegeneration, and immunization. ${ }^{10,13-16}$ PDIA4, one of the largest PDI members, comprises 645 amino acids and three classical CGHC active motifs. Similar to other PDI members, PDIA4 initiates coagulation and enhances thrombus formation via a series of cascade reactions..$^{17}$ In addition to the classic biological functions of PDIA4, emerging evidence indicates a potential association between PDIA4 and tumor development. ${ }^{17}$ Upregulated expression of PDIA4 was detected in a variety of tumor cell lines and in human lung adenocarcinoma tissue. PDIA4 expression mediates the inhibition of mitochondrial apoptosis-induced tumor death. ${ }^{18}$ Further, a study revealed that PDIA4 promotes tumor progression through the reduction of caspases $3 / 7 .{ }^{12}$ The ectopic expression and function of PDIA4 have also been reported in ovarian cancer. In ovarian carcinoma, PDIA4 was found as to take part in the drug-resistance phenotype and serve as a critical prognostic marker. ${ }^{19,20}$ Moreover, in pancreatic carcinoma, hepatocellular carcinoma, and esophageal squamous cell carcinoma, the increased expression of PDIA4 was associated with tumor development. ${ }^{21-25}$ In our previous studies, we have already described PDIA4 as one of the prognostic markers in lower-grade gliomas and its association with immunosuppressive TME. $^{26}$ From this perspective, we designed further experiments to study the molecular mechanisms and novel pathophysiological behaviors of PDIA4 in gliomas.

\section{Materials and Methods Data Sets}

The patient clinical annotation and gene expression data used in this study were obtained from publicly available databases. The TCGA lower grade glioma and glioblastoma (GBM; LGG) dataset, which included genomic and phenotypic data, were obtained from the University of California, Santa Cruz, Xena browser (https://xenabrow

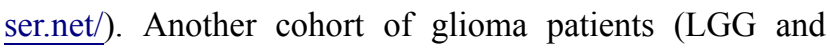
GBM) was obtained from the Chinese Glioma Genome Atlas (CGGA, http://www.cgga.org.cn/) and the mRNA sequencing data (RSEM) and clinical data were downloaded.

\section{Differential Expression Analysis}

Gene Expression Profiling Interactive Analysis (GEPIA) is an interactive web platform for gene expression analysis, which includes 9736 tumors and 8587 normal samples from TCGA and GTEx databases. Its gene expression data have been re-computed from raw RNA-Seq data based on the UCSC Xena project and a uniform pipeline for solving the imbalance between tumor and normal data. ${ }^{27}$ Differential expression analysis of PDIA4 between gliomas and normal brain tissues was performed using GEPIA.

\section{Survival Analysis}

Kaplan-Meier survival analysis and the Cox proportional hazard model were used to estimate the prognostic value of PDIA4 based on TCGA and CGGA datasets using $\mathrm{R}$ language packages (survival and survminer).

\section{Gene Ontology (GO) Enrichment Analysis}

The functional enrichment analysis, including gene ontology (GO) analysis comprised of cellular component (CC), molecular function (MF), biological process (BP), and Kyoto Encyclopedia of Genes and Genomes (KEGG) pathway, were performed via the cluster Profiler package in $\mathrm{R}$ language. ${ }^{28}$ Enriched ontological terms with an adjusted $\mathrm{P}$ value $<0.05$ were regarded as statistically significant.

\section{Analysis of Stromal and Immune Infiltration}

Stromal and immune infiltration was analyzes as described previously. ${ }^{29}$ The scores, calculated by the ESTIMATE 
algorithm, ${ }^{30}$ were downloaded from https://bioinformatics. mdanderson.org/estimate/. The pre-calculated TCGA data based on $\mathrm{xCell}^{31}$ was downloaded from http://xcell.ucsf. edu/. The correlation between PDIA4 expression and ESTIMATE scores and 64 cell types from the TCGA glioma dataset were then analyzed using $\mathrm{R}$ language.

\section{Protein-Protein Interaction (PPI) Analysis} The Search Tool for the Retrieval of Interacting Genes, ${ }^{32}$ an online database, was used to identify proteins that can interact with PDIA4 and construct PPI networks.

\section{Cell Lines and Culture}

All the cell lines were purchased from ATCC. The human glioma cell-lines (U87, U251, and T98G) and the normal glial cell-line HEB were cultured in DMEM with $10 \%$ FBS and antibiotics $(100 \mu \mathrm{g} / \mathrm{mL}$ penicillin and $100 \mu \mathrm{g} / \mathrm{mL}$ streptomycin), and maintained under standard culture conditions.

\section{RNA Extraction and Real-Time RT-PCR}

Total RNA was extracted from cell lines or human tissues using TRIzol reagent (Invitrogen) according to the manufacturer's protocol. Total RNA was quantified and $1 \mu \mathrm{g}$ of RNA was reverse-transcribed with the Reverse Transcription Kit (Thermo Fisher Scientific). qPCR was performed using SYBR Premix Ex Taq II (Takara Bio). $\beta$ actin mRNA was used to normalize gene expression. The primers used were as follows:

PDIA4: F: 5'- GGCAGGCTGTAGACTACGAG-3' and R: 5'- TTGGTCAACACAAGCGTGACT-3'

GAPDH: F: 5'-GGGAGCCAAAAGGGTCAT-3' and R: 5'-GTCCTTCCACGATACCAA-3'.

\section{PDIA4 shRNA Gene Silencing}

U87 and U251 cells were transfected with lentiviruses harboring PDIA4 shRNA (CTTGGTCCTAAATGATGCAAA) and control shRNA. Prior to use, shRNA-positive cells were validated by green fluorescence microscopy and selected for culturing in medium containing $2 \mu \mathrm{g} / \mathrm{mL}$ puromycin for 1 week.

\section{Western Blotting}

Cells were lysed with in RIPA buffer containing protease inhibitors. Equal amounts of protein samples were separated electrophoretically and then transferred onto PVDF membranes (Bio-Rad, Hercules, CA, USA). The membranes were blocked for $1 \mathrm{~h}$ in Tris-buffered saline containing Tween-20 (TBST) and 5\% non-fat milk. Thereafter,
Western blot analysis was performed using primary antibodies against PDIA4 (1:1000, Bioss ANTIBODIES, bs13106R), and $\beta$-actin in a blocking buffer containing 5\% non-fat milk and $0.1 \%$ Tween-20 in TBS. The blots were then developed using Lumiglo substrate (KP Laboratories, Gaithersburg, MD) on BioMax LS film (Eastman Kodak, Rochester, NY).

\section{IHC Staining}

Paraffin-embedded human glioma samples taken from glioma patients in Xiangya Hospital were resected. All patients signed informed consent to participate before taking samples. The cohort consisted of 15 cases, including 4, 5 , and 6 cases of WHO grade II-IV glioma tissues. The tumor tissues were formalin-fixed, processed, and paraffinembedded. Antigens were retrieved by autoclaving in 0.01 $\mathrm{mol} / \mathrm{L}$ sodium citrate buffer $(\mathrm{pH} 6.0)$ at $121^{\circ} \mathrm{C}$ and $20 \mathrm{psi}$ for 3-5 min. Endogenous peroxidase activity and nonspecific antibody-binding sites were blocked using 3\% hydrogen peroxide and $5 \%$ goat serum, respectively. The blocked sections were incubated overnight at $4{ }^{\circ} \mathrm{C}$ with primary antibody followed by $30 \mathrm{~min}$ incubation with secondary antibody. The slides were stained with diaminobenzidine (DAB) for $2 \mathrm{~min}$ and counterstained with hematoxylin. Scoring was performed considering the average percentage of positively stained cells counted in ten randomly selected visual fields. IHC was performed with primary antibodies against PDIA4 (1:100, Bioss ANTIBODIES, bs-13106R). IHC staining and quantification were performed by two blinded individuals.

\section{CCK8}

Proliferation in GBM cells was measured by Cell Counting kit-8 (Sigma-Aldrich, Shanghai, China), according to the manufacturer's instructions. Briefly, cells were seeded at a density of 3000 cells per well in 96-well plates. At each desired time point, CCK-8 solution was added (10 $\mu \mathrm{L} /$ well) to all wells and incubated for $2 \mathrm{~h}$ at $37^{\circ} \mathrm{C}$, followed by measurement of absorbance at $420 \mathrm{~nm}$ in a microplate reader (Model 680 microplate reader, BioRad Laboratories). Each experiment was performed in five replicates.

\section{Statistical Analysis}

Several packages (ggplot2, survival, survminer, corrplot) in the statistical software environment $\mathrm{R}$, version 3.5.3 (http://www.r-project.org) were used for statistical computations and for creating figures. Student's $t$-test and one- 
way ANOVA were used to analyze two or more groups of measurement data; Pearson's $r$ was used for correlation analysis. All the data were statistically significant $(\mathrm{P}<$ 0.05). The experiment was repeated three times.

\section{Results}

Association of PDIA4 with

\section{Clinicopathological Characters in}

\section{Gliomas}

In accordance with the hypothesis that PDIA4 plays a critical role in glioma aggressiveness, we first measured the expression of PDIA4 in glioma tissues compared to normal brain tissues. The remarkably increased expression of PDIA4 was observed in both GBM samples and lowgrade glioma (LGG) samples (Figure $1 \mathrm{~A}, \mathrm{p}<0.001$ ). To further validate this result, we performed q-PCR in glioma cell lines. The results showed that the mRNA expression of PDIA4 was elevated in glioma cell lines when compared with normal glial cell lines (Figure 1B). To further determine the expression of PDIA4 in glioma, we measured the expression level of PDIA4 in glioma tumor tissues via IHC staining. Resected glioma samples (Grade II-IV) were obtained from Xiangya Hospital and stained by PDIA4 primary antibody. We found a significant increase in the staining intensity in grade IV glioma samples, compared to the lower grade of tumors (Figure 1C and D). Similarly, PDIA4 expression positively correlated with glioma histological grade in both TCGA and CGGA cohorts (Figure 1E). It is widely recognized that mutations in isocitrate dehydrogenase genes (IDH1 and IDH2) have strong connections with tumor behaviors in gliomas. Patients with IDH-mutant (IDH-Mut) histology exhibited better prognosis than IDH-wildtype (IDHWt). Intriguingly, we also observed elevated expression levels of PDIA4 in the IDH-Wt subtype of glioma when compared with IDH-Mut tumors (Figure 1F). Additionally, we evaluated the expression of PDIA4 in different glioma patterns. The results showed higher expression of PDIA4 in mesenchymal and classical subtypes rather than neural and proneural subtypes (Figure 1G).

\section{The Expression of PDIA4 is Correlated with Prognosis in Glioma Patients}

Based on the findings that PDIA4 was aberrantly expressed in gliomas and showed strong relationships with histological grade and specific molecular subtype, we further studied the prognostic value of PDIA4 by
Kaplan-Meier survival analysis using data obtained from TCGA and CGGA datasets. The results indicated that high PDIA4 expression consistently correlated with poor patient outcomes in both the GBM group (Figure 2A and B) and LGG group (Figure 2C and D). To study whether PDIA4 is an independent prognostic factor in glioma, we also performed Cox regression analysis with data obtained from TCGA and CGGA. In multivariate analysis, after adjusting for many clinical factors, such as patient age, patient sex, WHO grade, and IDH status, the results suggested that the expression of PDIA4 was a strong predictor in patients with glioma (Tables 1 and 2).

\section{Functional Enrichment of PDIA4 in Glioma}

To understand the mechanism by which PDIA4 promotes tumor growth and illustrates the key signaling regulated by PDIA4, we performed GO functional enrichment analysis.

Data from both TCGA and CGGA were analyzed by Pearson correlation analysis and genes with $|\mathrm{R}|>0.6$ were collected for functional enrichment. As a consequence, 408 terms of biological process (BP), 110 terms of cellular component (CC), 40 terms of molecular function (MF) were identified from TCGA database, and 140 terms of BP, 56 terms of CC, and 15 terms of MF were identified from the CGGA database (Supplementary Tables 1 and 2). The top 10 terms of BP mainly enriched functions of neutrophil-mediated immune function (Figure $3 \mathrm{~A}$ and $\mathrm{B}$ ). MF enrichment indicated functions predominantly involved in transferase activities, cell adhesion, and molecule binding (Figure 3C and D). Meanwhile, genes from CC terms were significantly associated with focal adhesion, cell-substrate junctions, and endoplasmic reticulum lumen (Figure 3E and F). We also conducted KEGG pathway analysis with selected genes. The results revealed a strong correlation between PDIA4 related genes and important biological signaling, such as protein processing in the endoplasmic reticulum, human immunodeficiency virus 1 infection, and apoptosis (Figure 3G and $\mathrm{H}$ ).

\section{PDIA4 Correlates with the TME and Promotes the Viability of Glioma Cells}

Considering the results from GO and KEGG pathway analysis, which elucidated strong connections between PDIA4 and immunological functions, we next performed examinations to confirm this phenomenon. First, we examined the association between PDIA4 expression and 
A

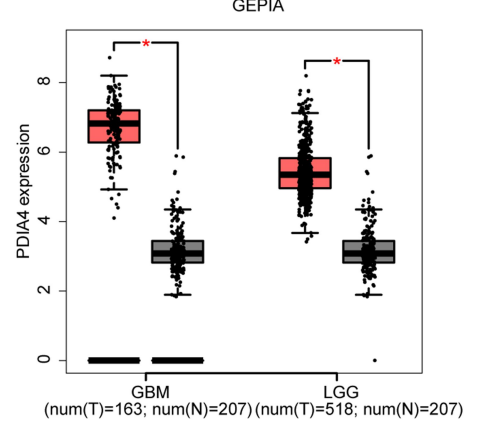

C

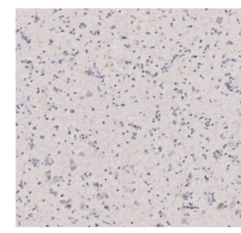

Control

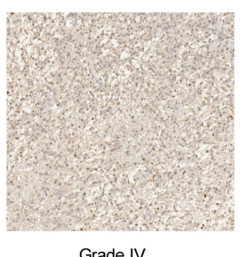

Grade IV

E

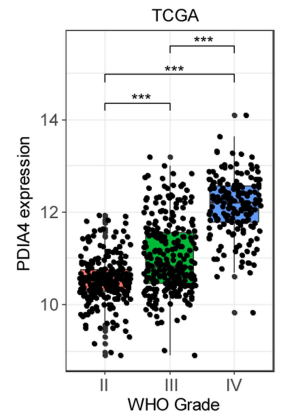

B

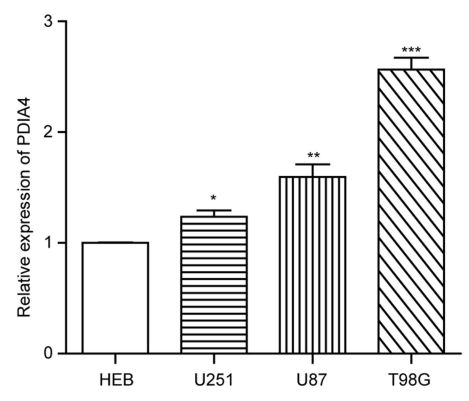

D

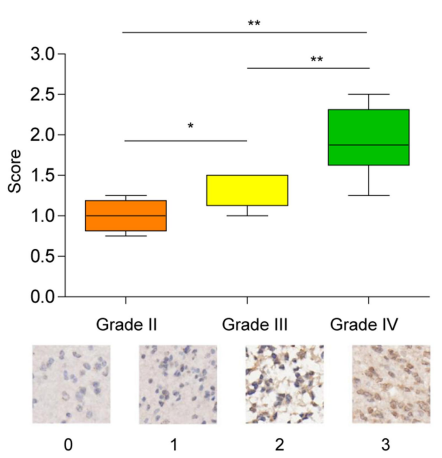

F

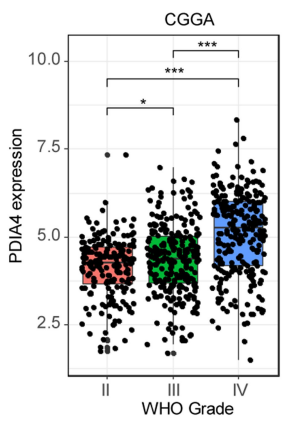

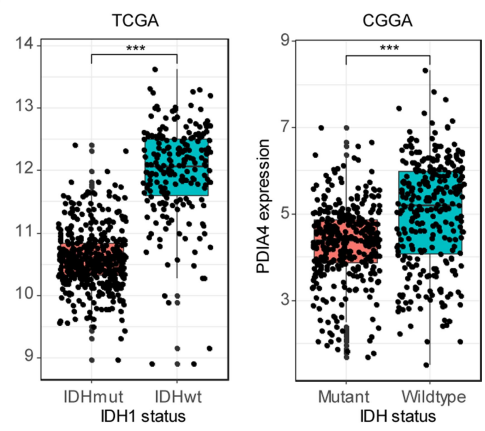

G

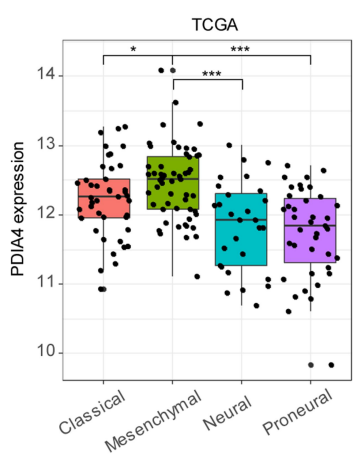

Figure I PDIA4 is highly expressed in gliomas and significantly associated with the advanced stage of tumors. (A) Differential expression of LCTL in brain lower grade glioma (LGG) and glioblastoma (GBM) compared to levels in normal brain tissues. ${ }^{*} \mathrm{P}<0.05$. (B) Relative mRNA expression level of PDIA4 in the normal glial cell line HEB and glioma cell lines. $* \mathrm{P}<0.05$; **P $<0.01$; ***P $<0.00 \mathrm{I}$. (C) IHC staining of PDIA4 in WHO grade IV gliomas of and comparison with normal brain tissues resected at Xiangya Hospital. Original magnification, $\times 100$. Scale bar, 50 glioma tissue arrays. The lower section shows the representative intensity at each level of PDIA4 IHC staining. *P $<0.05$; **P $<0.01$. (E) PDIA4 expression in gliomas of WHO grade III-V, based on both TCGA and CGGA datasets. *P $<0.05$; ***P $<0.00 \mathrm{I}$. (F) The expression of PDIA4 in IDH subtypes of gliomas based on both the TCGA and CGGA datasets. ***P $<0.00 \mathrm{I}$. (G) PDIA4 expression pattern in different molecular subtypes of glioma (classical, mesenchymal, neural, proneural) in the TCGA dataset. $* \mathrm{P}<0.05 ; * * * \mathrm{P}<0.001$. 
survival analysis of PDAI4 in GBM patients

A

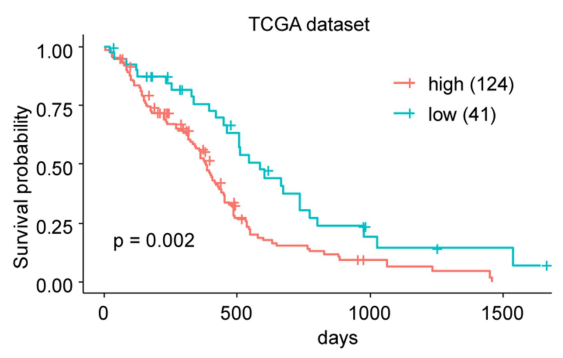

B

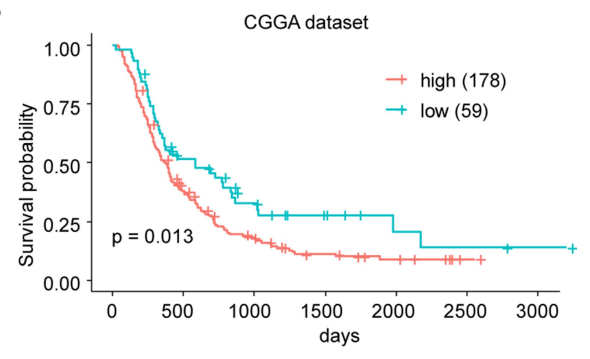

survival analysis of PDAI4 in LrGG patients

C

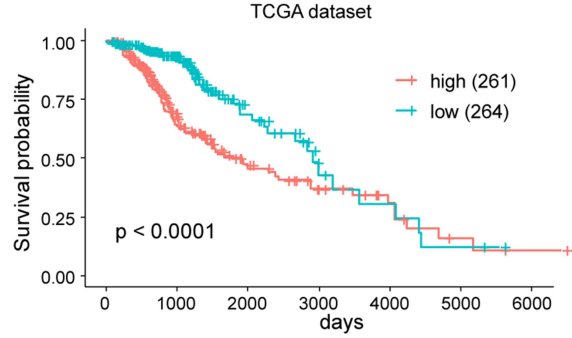

D

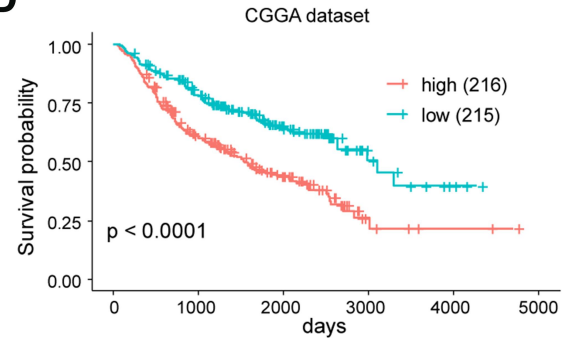

Figure 2 PDIA4 is a prognostic factor for glioma patients. (A and B) Kaplan-Meier survival analysis showing high PDIA4 expression predicts poor prognosis for glioblastoma multiform (GBM) patients in both the TCGA and CGGA datasets. The upper quartile number was used in this analysis. (C and D) Kaplan-Meier survival analysis showing that high PDIA4 expression predicts poor prognosis for lower grade glioma (LGG) patients in both the TCGA and CGGA datasets. The median number was used in this analysis.

immune scores. The results showed that PDIA4 expression had a relatively lower correlation with both stromal score and immune score in GBM patients (Figure 4A). However, in LGG samples, we found a strong correlation between PDIA4 and stromal or immune scores (Figure 4B).
Moreover, we studied the correlation between PDIA4 and 64 non-cancerous cell types to determine the critical cellular components involved in PDIA4 associated immunological processes. The results revealed that 46 cell types correlated with PDIA4, among which 33 types were

Table I Univariate and Multivariate Analysis Based on the TCGA Dataset

\begin{tabular}{|l|l|l|l|l|}
\hline \multirow{2}{*}{ Variable } & \multicolumn{2}{|l|}{ Univariate Analysis } & \multicolumn{2}{l|}{ Multivariate Analysis } \\
\cline { 2 - 5 } & HR (95\% CI) & $\mathbf{P}$ & HR (95\% CI) & $\mathbf{P}$ \\
\hline PDIA4 & $3.000(2.608-3.452)$ & $<0.001$ & $1.304(1.007-1.689)$ & $<0.05$ \\
Gender & $0.8 I 7(0.637-1.049)$ & $>0.05$ & $0.758(0.572-1.004)$ & $>0.05$ \\
Age & $1.068(1.058-1.078)$ & $<0.001$ & $1.036(1.024-1.049)$ & $<0.001$ \\
WHO grade & $9.524(7.230-12.550)$ & $<0.001$ & $2.021(1.368-2.986)$ & $<0.01$ \\
IDH status & $10.010(7.44 I-13.470)$ & $<0.001$ & $3.477(2.110-5.729)$ & $<0.001$ \\
\hline
\end{tabular}

Table 2 Univariate and Multivariate Analysis Based on the CCGA Dataset

\begin{tabular}{|l|l|l|l|l|}
\hline \multirow{2}{*}{ Variable } & \multicolumn{2}{l|}{ Univariate Analysis } & \multicolumn{2}{l|}{ Multivariate Analysis } \\
\cline { 2 - 5 } & HR (95\% CI) & $\mathbf{P}$ & HR (95\% CI) & $\mathbf{P}$ \\
\hline PDIA4 & $1.618(1.477-1.773)$ & $<0.001$ & $1.27 I(1.149-1.406)$ & $<0.001$ \\
Gender & $1.012(0.826-1.24 I)$ & $>0.05$ & $0.977(0.793-1.205)$ & $>0.05$ \\
Age & $1.027(1.018-1.035)$ & $<0.001$ & $1.011(1.002-1.019)$ & $<0.05$ \\
WHO grade & $3.979(3.227-4.906)$ & $<0.001$ & $2.142(1.621-2.831)$ & $<0.001$ \\
IDH status & $3.238(2.616-4.009)$ & $<0.001$ & $1.846(1.422-2.397)$ & $<0.001$ \\
\hline
\end{tabular}


PDIA4 related BP terms

A

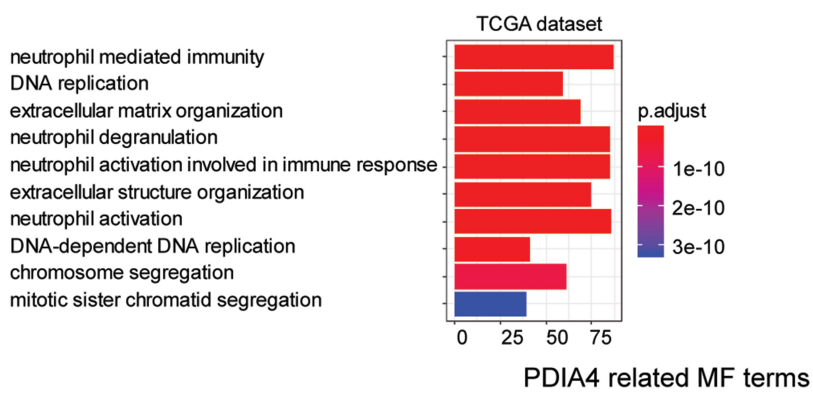

B

neutrophil mediated immunity protein folding neutrophil degranulation neutrophil activation involved in immune response glycoprotein biosynthetic process neutrophil activation

response to topologically incorrect protein glycoprotein metabolic process response to unfolded protein IRE1-mediated unfolded protein response

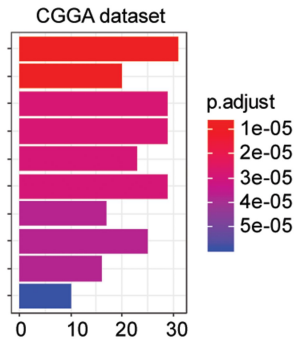

C

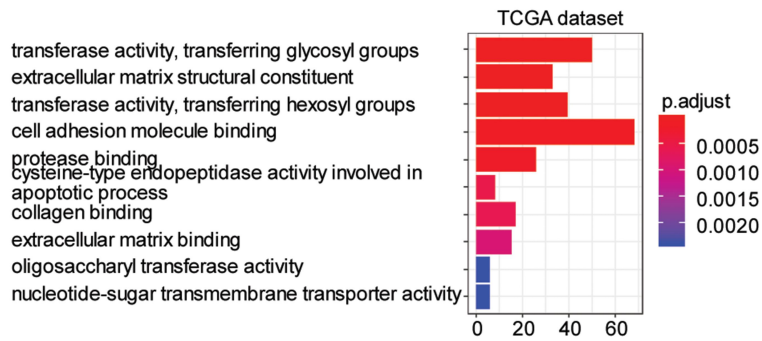

D

transferase activity, transferring hexosyl groups
transferase activity, transferring glycosyl groups
oligosaccharyl transferase activity
isomerase activity
cysteine-type endopeptidase activity involved in
apoptotic process
death receptor binding
cell adhesion molecule binding
disulfide oxidoreductase activity
cadherin binding
intramolecular oxidoreductase activity

CGGA dataset

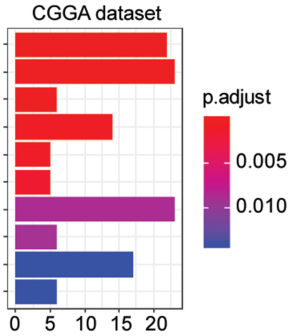

PDIA4 related $\mathrm{CC}$ terms

E

focal adhesion
cell-substrate adherens junction
cell-substrate junction
endoplasmic reticulum lumen
collagen-containing extracellular matrix
chromosomal region
condensed chromosome
condensed chromosome, centromeric region
extracellular matrix
chromosome, centromeric region

G

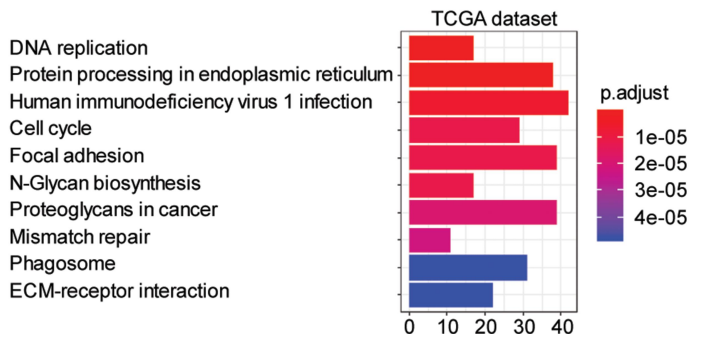

$\mathbf{F}$

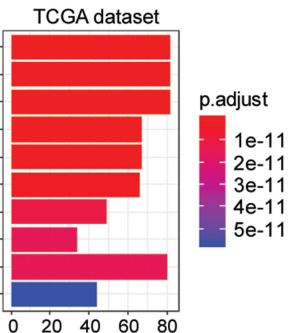

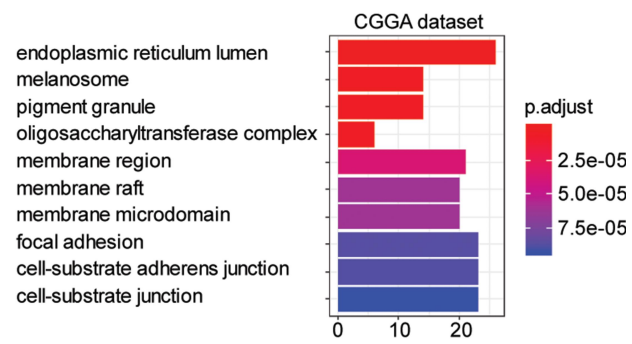

PDIA4 related KEGG pathways

H

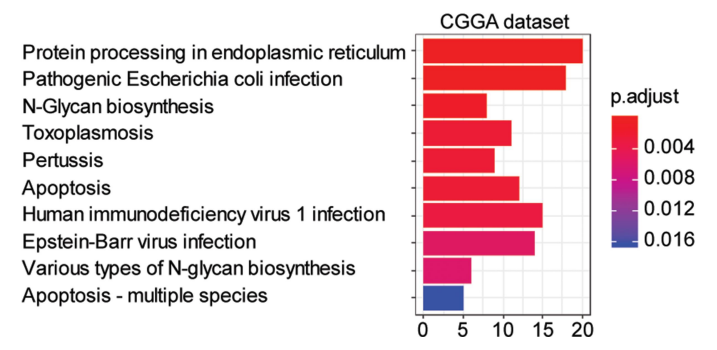

Figure 3 Functional enrichment analysis of PDIA4 in TCGA and CGGA cohorts. Data of all grades of glioma were used in this analysis. (A and B) The top 10 biological process terms of GO enrichment analysis based on TCGA and CGGA datasets, respectively. (C and D) The top 10 molecular function terms of GO enrichment analysis based on TCGA and CGGA datasets, respectively. (E and F) The top 10 cellular component terms of GO enrichment analysis based on TCGA and CGGA datasets, respectively. (G and $\mathbf{H})$ KEGG pathway analysis based on TCGA and CGGA datasets and the top 10 terms were visualized.

positively related, whereas 13 types were negatively related (Figure 4C, Table 3). Notably, the cellular components that exhibited a dramatic correlation with PDIA4, such as astrocytes, M1 macrophages, $\mathrm{CD} 4^{+}$memory $\mathrm{T}$ cells, $\mathrm{CD} 8^{+} \mathrm{T}$ cells, Tregs, and eosinophils, have already been demonstrated to play critical roles in the glioma TME. We further validated the correlation between
PDIA4 and immune properties via classic immunological markers. The results suggested that PDIA4 was closely related to several immunosuppressive factors, especially the dendritic cells, M2 macrophages, monocytes, and T cell exhaustion markers (Table 4). Besides, we subjected PDIA4 to protein interaction analysis (PPI) to study the regulatory network of this protein. Based on these results, 
A

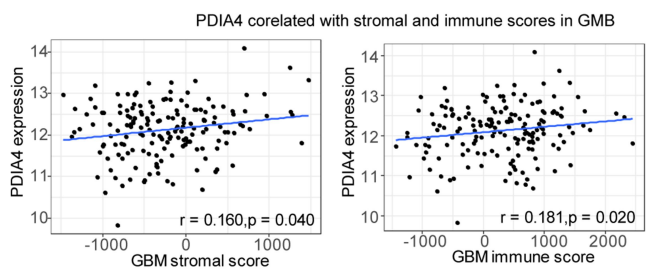

B

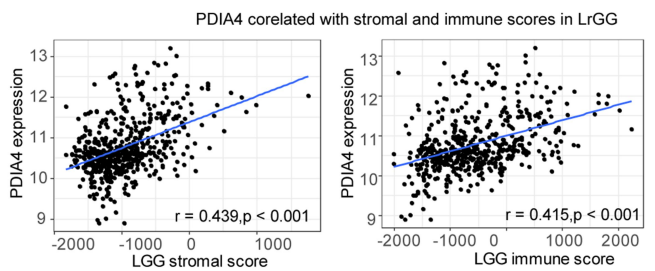

D

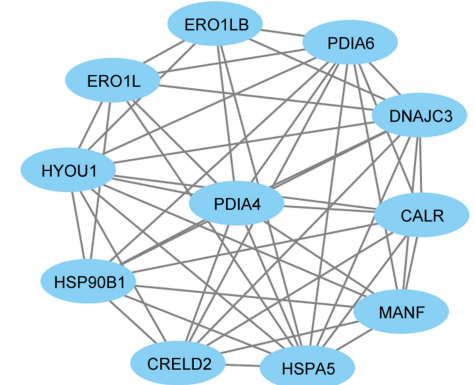

E

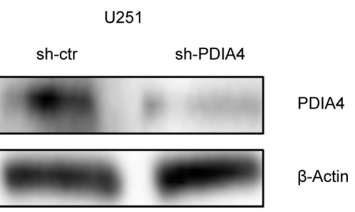

C

PDIA4 significantly corelated with 46 cell types

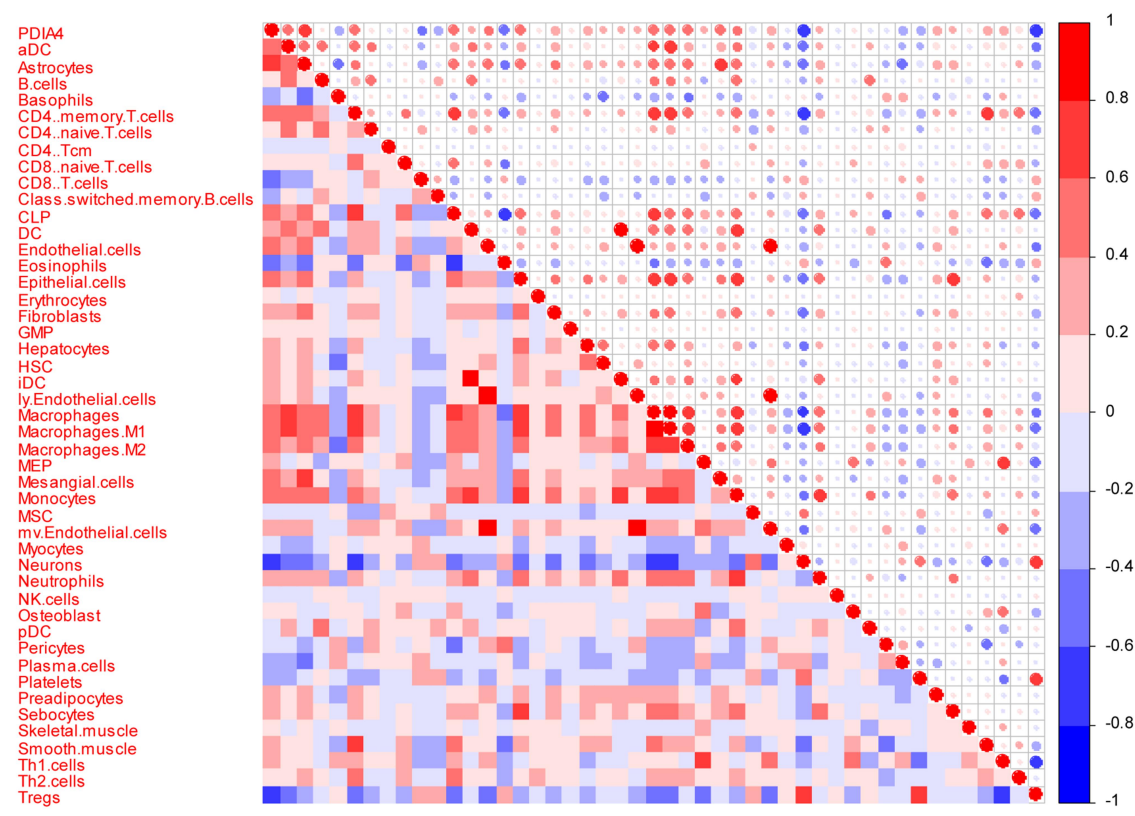

F

$\mp$ sh-ctr $\rightarrow$ sh-PDIA4

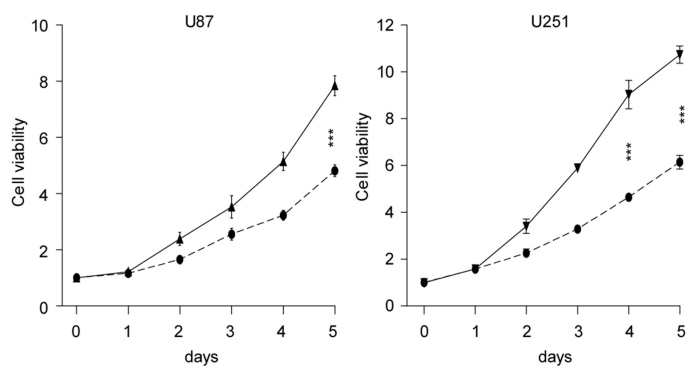

Figure 4 PDIA4 correlates with TME and promotes glioma cell proliferation.(A) PDIA4 expression positively correlated with immune score and stromal score in glioblastoma multiform (GBM) patients. (B) PDIA4 expression positively correlated with immune score and stromal score in lower grade glioma (LGG) patients. (C) PDIA4 expression significantly correlated with 46 cell types, as calculated by xCells in glioma. Data of all grades of glioma were used in this analysis. (D) Protein-protein interaction (PPI) network of PDIA4. Data of all grades of glioma were used in this analysis. (E) Demonstration of PDIA4 knockdown (KD) in U87 and U25I GBM cells by Western blotting analysis. Cells transfected with non-specific shRNA were used as control. (F) A total of 3000 control and PDIA4 KD glioma cells were plated in a 96 -well plate in $200 \mu \mathrm{L}$ medium. Cell viability was assayed using CCK8 assay. Both PDIA4 KD U87 and U25I cells showed significantly reduced cell viability compared to control. ***P $<0.00$ I. 
Table 3 Correlation Ship Between PDIA4 and 64 Types of Non-Cancerous Cells

\begin{tabular}{|c|c|c|c|}
\hline xCells & Category & Pearson's r $(95 \% \mathrm{Cl})$ & adj.p \\
\hline B cells & lymphoids & $0.089(-0.042 \sim 0.217)$ & * \\
\hline CD4+ memory $T$ cells & lymphoids & $0.54 \mathrm{I}(0.442 \sim 0.627)$ & $* * *$ \\
\hline CD4+ naive $T$ cells & lymphoids & $0.175(0.045 \sim 0.298)$ & $* * *$ \\
\hline CD4+ $T$ cells & lymphoids & $-0.021(-0.151 \sim 0.109)$ & \\
\hline $\mathrm{CD} 4+\mathrm{Tcm}$ & lymphoids & $-0.091(-0.219 \sim 0.04)$ & * \\
\hline CD4+Tem & lymphoids & $0.054(-0.076 \sim 0.183)$ & \\
\hline CD8+ naive $T$ cells & lymphoids & $0.127(-0.004 \sim 0.253)$ & $* *$ \\
\hline $\mathrm{CD} 8+\mathrm{Tcm}$ & lymphoids & $-0.413(-0.515 \sim-0.299)$ & $* * *$ \\
\hline CD8+ Tem & lymphoids & $0.055(-0.075 \sim 0.184)$ & \\
\hline $\mathrm{CD} 8+\mathrm{T}$ cells & lymphoids & $0.062(-0.069 \sim 0.19)$ & \\
\hline Class switched memory B cells & lymphoids & $-0.295(-0.409 \sim-0.171)$ & $* * *$ \\
\hline Memory B cells & lymphoids & $0.003(-0.127 \sim 0.133)$ & \\
\hline naive $B$ cells & lymphoids & $-0.002(-0.132 \sim 0.129)$ & \\
\hline NK cells & lymphoids & $-0.094(-0.222 \sim 0.036)$ & * \\
\hline Natural killer T cells (NKT) & lymphoids & $0.043(-0.088 \sim 0.172)$ & \\
\hline Plasma cells & lymphoids & $-0.359(-0.467 \sim-0.24)$ & $* * *$ \\
\hline pro B cells & lymphoids & $0.006(-0.124 \sim 0.136)$ & \\
\hline Tgd cells & lymphoids & $0.038(-0.093 \sim 0.168)$ & \\
\hline ThI cells & lymphoids & $0.394(0.278 \sim 0.499)$ & $* * *$ \\
\hline Th2 cells & lymphoids & $0.198(0.07 \sim 0.32)$ & $* * *$ \\
\hline Tregs & lymphoids & $-0.627(-0.7 \sim-0.54 \mathrm{I})$ & $* * *$ \\
\hline Activated dendritic cells (aDC) & myeloids & $0.468(0.36 \sim 0.564)$ & $* * *$ \\
\hline Basophils & myeloids & $-0.325(-0.437 \sim-0.204)$ & $* * *$ \\
\hline Conventional dendritic cells (cDC) & myeloids & $0.02(-0.111 \sim 0.149)$ & \\
\hline Dendritic cells (DC) & myeloids & $0.234(0.107 \sim 0.354)$ & $* * *$ \\
\hline Eosinophils & myeloids & $-0.491(-0.584 \sim-0.385)$ & $* * *$ \\
\hline Immature DC (iDC) & myeloids & $0.202(0.074 \sim 0.324)$ & $* * *$ \\
\hline Macrophages y & myeloids & $0.55 \mathrm{I}(0.454 \sim 0.636)$ & $* * *$ \\
\hline Macrophages MI & myeloids & $0.593(0.501 \sim 0.671)$ & $* * *$ \\
\hline Macrophages M2 & myeloids & $0.44 \mathrm{I}(0.329 \sim 0.54)$ & $* * *$ \\
\hline Mast cells & myeloids & $0.061(-0.07 \sim 0.189)$ & \\
\hline Monocytes & myeloids & $0.40 \mathrm{I}(0.286 \sim 0.505)$ & $* * *$ \\
\hline Neutrophils & myeloids & $0.286(0.162 \sim 0.401)$ & $* * *$ \\
\hline Plasmacytoid dendritic cells (pDC) & myeloids & $-0.093(-0.22 \sim 0.038)$ & * \\
\hline Astrocytes & others & $0.767(0.707 \sim 0.815)$ & $* * *$ \\
\hline Epithelial cells & others & $0.517(0.415 \sim 0.607)$ & $* * *$ \\
\hline Hepatocytes & others & $0.33 \mathrm{I}(0.21 \sim 0.442)$ & $* * *$ \\
\hline Keratinocytes & others & $0.064(-0.067 \sim 0.193)$ & \\
\hline Melanocytes & others & $0.002(-0.128 \sim 0.133)$ & \\
\hline Mesangial cells & others & $0.505(0.401 \sim 0.596)$ & $* * *$ \\
\hline Myocytes & others & $-0.163(-0.287 \sim-0.033)$ & $* * *$ \\
\hline Neurons & others & $-0.687(-0.75 \sim-0.612)$ & $* * *$ \\
\hline Sebocytes & others & $0.273(0.148 \sim 0.389)$ & $* * *$ \\
\hline Common lymphoid progenitors (CLP) & stem cells & $0.565(0.47 \sim 0.648)$ & $* * *$ \\
\hline Common myeloid progenitors (CMP) & stem cells & $0.007(-0.123 \sim 0.137)$ & \\
\hline Erythrocytes & stem cells & $0.092(-0.038 \sim 0.22)$ & * \\
\hline Granulocyte-macrophage progenitor (GMP) & stem cells & $0.138(0.008 \sim 0.264)$ & $* * *$ \\
\hline Hematopoietic stem cells (HSC) & stem cells & $0.283(0.158 \sim 0.398)$ & $* * *$ \\
\hline Megakaryocytes & stem cells & $-0.01(-0.14 \sim 0.121)$ & \\
\hline Megakaryocyte-erythroid progenitors (MEP) & stem cells & $0.273(0.148 \sim 0.39)$ & $* * *$ \\
\hline Multipotent progenitors (MPP) & stem cells & $-0.015(-0.145 \sim 0.116)$ & \\
\hline Platelets & stem cells & $-0.399(-0.503 \sim-0.283)$ & $* * *$ \\
\hline Adipocytes & stromal cells & $-0.01(-0.141 \sim 0.12)$ & \\
\hline Chondrocytes & stromal cells & $0.026(-0.104 \sim 0.156)$ & \\
\hline
\end{tabular}

(Continued) 
Table 3 (Continued).

\begin{tabular}{|c|c|c|c|}
\hline xCells & Category & Pearson's r (95\% Cl) & adj.p \\
\hline Endothelial cells & stromal cells & $0.412(0.297 \sim 0.514)$ & $* * *$ \\
\hline Fibroblasts & stromal cells & $0.376(0.259 \sim 0.483)$ & $* * *$ \\
\hline ly Endothelial cells & stromal cells & $0.248(0.122 \sim 0.366)$ & $* * *$ \\
\hline Mesenchymal stem cells (MSC) & stromal cells & $-0.196(-0.318 \sim-0.067)$ & **** \\
\hline mv Endothelial cells & stromal cells & $0.333(0.212 \sim 0.444)$ & $* * *$ \\
\hline Osteoblast & stromal cells & $0.108(-0.022 \sim 0.235)$ & $* *$ \\
\hline Pericytes & stromal cells & $-0.171(-0.295 \sim-0.042)$ & $* * *$ \\
\hline Preadipocytes & stromal cells & $0.385(0.268 \sim 0.49)$ & $* * *$ \\
\hline Skeletal muscle & stromal cells & $0.128(-0.002 \sim 0.254)$ & $* *$ \\
\hline Smooth muscle & stromal cells & $0.393(0.277 \sim 0.498)$ & **** \\
\hline
\end{tabular}

Notes: $* \mathrm{P}<0.05 ; * * \mathrm{P}<0.01 ; * * * \mathrm{P}<0.001$.

we found that PDIA4 interacts with several heat shock proteins (Figure 4D). Functional studies revealed that these genes were closely related to stress-reduced responses and endoplasmic reticulum, which was consistent with the data described above. Meanwhile, the majority of PDIA4-related genes, such as PDIA6, ERO1LB, ERO1L, HSPA5, HSP90B1, and HYOU1, were found to be tightly involved in the tumor-promoting phenotype. To explore the role of PDIA4 in GBM tumorigenesis, we established PDIA4 knockdown clones of U87 and U251 glioma cells with specific shRNA. The efficiency of PDIA4 knockdown was confirmed by Western blotting and selected for the next CCK8 experiments (Figure 4E). We found that knockdown of PDIA4 significantly inhibited the proliferative activities compared to control cells (Figure 4F).

\section{Discussion}

Despite the current multi-therapeutic strategies against glioma, including modern neurosurgery, radiotherapy, chemotherapy, and immunotherapy, the prognosis of glioma patients remains poor because of the aggressive features of this type of cancer. Novel, efficient management of glioma requires a comprehensive understanding of the biological nature of this disease. The illustration of potential critical factors that overexpress and play an essential role in glioma progression is of great importance to increase our knowledge of this malignant disease. Our present study first identified PDIA4 as a novel molecular marker that shows a close relationship with the clinicopathological characteristics and immunological surveillance of glioma, and provides alternative strategies for the subsequent treatment of this disease.
PDIA4 was originally described to be involved in various biological processes, including coagulation, ${ }^{33}$ thrombosis formation ${ }^{34,35}$ and injury reaction. ${ }^{36}$ Recently, there has been a mounting evidence that aberrant expression and the potential mechanisms of PDIA4 participate in the development of multiple types of cancer. ${ }^{12,17,18,21}$ Moreover, our recent study documented that PDIA4 is involved in the prognostic model in LGG, subsequently participating in the immunosuppressive TME. ${ }^{26}$ Based on these findings, the present study identified PDIA4 overexpression not only in glioma tissues but also its significant consistency with WHO grade. In addition, our study revealed increased mRNA expression of PDIA4 in glioma cell lines. Mechanistically, PDIA4 was significantly associated with the IDH status and different glioma subtypes. Furthermore, we examined the clinical importance of PDIA4 and found that PDIA4 was an independent prognostic marker whose expression was negatively correlated with the outcomes of glioma patients. To elucidate the critical functions of PDIA4 in glioma, we conducted GO function and KEGG pathway analysis in both TCGA and CGGA datasets. As a result, the PDIA4-related biological functions were mainly enriched in transferase activities, endoplasmic reticulum responses, and immunity.

The orchestrated immunological interactions within glioma TME have received increased attention, and harnessing the immune system is becoming a hotspot in the field of oncology. Various components of glioma TME, such as immune cells, cytokines, and markers, coordinately interact with each other to establish the immunosuppressive phenotype and promote the development of glioma. ${ }^{5}$ From this perspective, advanced clinical practices targeting specific immunotherapies have already shown profound outcomes compared to conventional therapy 
Table 4 Correlation Analysis Between LAYN and Relate Genes and Markers of Immune Cells Based on TCGA Database

\begin{tabular}{|c|c|c|c|}
\hline Description & Gene Markers & Pearson's r $(95 \% \mathrm{Cl})$ & $\mathbf{P}$ \\
\hline CD8+ $\mathrm{T}$ cell & $\begin{array}{l}\text { CD8A } \\
C D 8 B\end{array}$ & $\begin{array}{l}0.375(-0.062-0.692) \\
0.322(-0.123-0.659)\end{array}$ & $\begin{array}{l}* * \\
*\end{array}$ \\
\hline T cell (general) & $\begin{array}{l}C D 3 D \\
C D 3 E \\
C D 2\end{array}$ & $\begin{array}{l}0.332(-0.1 \mathrm{II}-0.665) \\
0.433(0.006-0.726) \\
0.427(-0.00 \mathrm{I}-0.723)\end{array}$ & $\begin{array}{l}* \\
* * \\
* *\end{array}$ \\
\hline B cell & $\begin{array}{l}\text { CD19 } \\
\text { CD79A }\end{array}$ & $\begin{array}{l}0.201(-0.248-0.579) \\
0.233(-0.216-0.601)\end{array}$ & \\
\hline Monocyte & $\begin{array}{l}\text { CD86 } \\
C D I / 5 \text { (CSFIR) }\end{array}$ & $\begin{array}{l}0.616(0.255-0.826) \\
0.617(0.257-0.826)\end{array}$ & $\begin{array}{l}* * * \\
* * *\end{array}$ \\
\hline TAM & $\begin{array}{l}\text { CCL2 } \\
\text { CD68 } \\
\text { ILIO }\end{array}$ & $\begin{array}{l}0.569(0.187-0.802) \\
0.806(0.577-0.917) \\
0.355(-0.086-0.68)\end{array}$ & $\begin{array}{l}* * * \\
* * * \\
*\end{array}$ \\
\hline MI macrophage & $\begin{array}{l}\text { INOS (NOS2) } \\
\text { IRF5 } \\
\text { COX2(PTGS2) }\end{array}$ & $\begin{array}{l}0.386(-0.05-0.698) \\
0.638(0.289-0.837) \\
0.354(-0.087-0.679)\end{array}$ & $\begin{array}{l}* * \\
* * * \\
*\end{array}$ \\
\hline M2 macrophage & $\begin{array}{l}\text { CDI } 63 \\
\text { VSIG4 } \\
\text { MS4A4A }\end{array}$ & $\begin{array}{l}0.555(0.167-0.794) \\
0.651(0.309-0.844) \\
0.66(0.324-0.848)\end{array}$ & $\begin{array}{l}* * * \\
* * * \\
* * *\end{array}$ \\
\hline Neutrophils & $\begin{array}{l}\text { CD66b (CEACAM8) } \\
\text { CD I Ib (ITGAM) } \\
\text { CCR7 }\end{array}$ & $\begin{array}{l}0.121(-0.323-0.522) \\
0.553(0.164-0.793) \\
0.34(-0.102-0.67)\end{array}$ & $\begin{array}{l}* * * \\
*\end{array}$ \\
\hline Natural killer cell & $\begin{array}{l}\text { KIR2DLI } \\
\text { KIR2DL3 } \\
\text { KIR2DL4 } \\
\text { KIR3DLI } \\
\text { KIR3DL2 } \\
\text { KIR3DL3 } \\
\text { KIR2DS4 }\end{array}$ & $\begin{array}{l}-0.039(-0.459-0.395) \\
-0.003(-0.43-0.425) \\
0.069(-0.369-0.483) \\
0.13(-0.315-0.528) \\
0.057(-0.38-0.473) \\
-0.374(-0.69 \mid-0.064) \\
0.05 I(-0.385-0.468)\end{array}$ & $* *$ \\
\hline Dendritic cell & $\begin{array}{l}\text { HLA-DPBI } \\
\text { HLA-DQBI } \\
\text { HLA-DRA } \\
\text { HLA-DPAI } \\
\text { BDCA-I(CDIC) } \\
\text { BDCA-4(NRPI) } \\
\text { CDIIC (ITGAX) }\end{array}$ & $\begin{array}{l}0.644(0.299-0.84) \\
0.549(0.159-0.791) \\
0.692(0.376-0.864) \\
0.652(0.311-0.844) \\
0.17(-0.278-0.557) \\
0.876(0.716-0.948) \\
0.529(0.131-0.78)\end{array}$ & $\begin{array}{l}* * * \\
* * * \\
* * * \\
* * * \\
* * * \\
* * *\end{array}$ \\
\hline ThI & $\begin{array}{l}\text { T-bet (TBX2I) } \\
\text { STAT4 } \\
\text { STATI } \\
\text { IFN-g (IFNG) } \\
\text { TNF-a (TNF) }\end{array}$ & $\begin{array}{l}0.372(-0.066-0.69) \\
0.081(-0.36-0.491) \\
0.822(0.608-0.925) \\
0.128(-0.317-0.526) \\
0.158(-0.289-0.549)\end{array}$ & $\begin{array}{l}* * \\
* * *\end{array}$ \\
\hline Th2 & $\begin{array}{l}\text { GATA3 } \\
\text { STAT6 } \\
\text { STAT5A } \\
\text { ILI3 }\end{array}$ & $\begin{array}{l}0.389(-0.047-0.7) \\
0.808(0.582-0.918) \\
0.735(0.449-0.885) \\
0.076(-0.364-0.488)\end{array}$ & $\begin{array}{l}* * \\
* * * \\
* * *\end{array}$ \\
\hline
\end{tabular}

(Continued) 
Table 4 (Continued).

\begin{tabular}{|c|c|c|c|}
\hline Description & Gene Markers & Pearson's r $(95 \% \mathrm{Cl})$ & $\mathbf{P}$ \\
\hline Tfh & $\begin{array}{l}\text { BCL6 } \\
\text { IL2I }\end{array}$ & $\begin{array}{l}0.703(0.394-0.869) \\
-0.154(-0.546-0.293)\end{array}$ & $* * *$ \\
\hline ThI7 & $\begin{array}{l}\text { STAT3 } \\
\text { ILI } 7 A\end{array}$ & $\begin{array}{l}0.896(0.759-0.957) \\
-0.083(-0.493-0.358)\end{array}$ & $* * *$ \\
\hline Treg & $\begin{array}{l}\text { FOXP3 } \\
\text { CCR8 } \\
\text { STAT5B } \\
\text { TGFb (TGFBI) }\end{array}$ & $\begin{array}{l}0.316(-0.129-0.655) \\
0.098(-0.344-0.505) \\
0.756(0.485-0.895) \\
0.8(0.567-0.915)\end{array}$ & $\begin{array}{l}* \\
* * * \\
* * *\end{array}$ \\
\hline T cell exhaustion & $\begin{array}{l}\text { PD-I (PDCDI) } \\
\text { CTLA4 } \\
\text { LAG3 } \\
\text { TIM-3 (HAVCR2) } \\
\text { GZMB }\end{array}$ & $\begin{array}{l}0.37(-0.068-0.689) \\
0.257(-0.192-0.617) \\
0.484(0.071-0.756) \\
0.648(0.305-0.842) \\
0.243(-0.206-0.607)\end{array}$ & $\begin{array}{l}* * \\
* * * \\
* * *\end{array}$ \\
\hline
\end{tabular}

Notes: $* \mathrm{P}<0.05 ; * * \mathrm{P}<0.01$; $* * * \mathrm{P}<0.001$.

against glioma. As PDIA4 was previously shown to participate in the immunological TME in LGG, we further detected its correlation with multiple immune factors. Consistent with our study, we found that PDIA4 was tightly related to both the immune and stromal scores in LGG. The relationship between PDIA4 and immune scores was relatively lower in GBM, suggesting the potential heterogeneities between different grades of glioma. After checking the association between PDIA4 and 64 non-cancerous cells, we found a significant link between 46 types of cells and PDIA4. Furthermore, we studied the connections between PDIA4 and classic genes and markers of immune cells. Interestingly, the data showed a close relationship with several infiltrating immune cells, such as monocytes, tumor-associated macrophages, and neutrophils, which are widely considered immunosuppressive components in glioma TME. An association has also been detected among dendritic cell markers and PDIA4, which suggested the potential functions of PDIA4 in the process of antigen presentation and immune surveillance. Consistently, our PPI analysis of PDIA4 indicated that the major proteins related to PDIA4 are members of heat shock proteins or endoplasmic reticulum proteins, which have also been reported to be tumor promoting in various cancers. ${ }^{21,37-40}$ Next, our phenotypic experiments showed that downregulation of PDIA4 significantly inhibited the proliferation of glioma cells, indicating that pharmacological inhibition of PDIA4 could be regarded as a novel therapeutic target against glioma.
Based on our findings, our study is the first to describe the novel function of PDIA4, and we propose a new linkage between PDIA4 and various immune components in glioma. PDIA4 is highly expressed in glioma and is significantly associated with clinical outcomes. The tumor-promoting characteristics of PDIA4 are potentially mediated by the immune system via certain connections with multiple immune factors in the glioma TME. The detailed molecular mechanism of PDIA4 and the development of glioma need to be further elucidated. To this end, our study provides novel possibilities for finding new therapeutic approaches targeting PDIA4 in glioma.

\section{Conclusion}

PDIA4 expression was high in glioma samples and associated with a potentially poor prognosis in glioma patients. Mechanistically, we demonstrated that PDIA4 has an immunosuppressive role and regulates the recruitment of immunosuppressive cells within the glioma tumor microenvironment. PDIA4 knockdown significantly impaired the proliferation of GBM cell lines. Therefore, we suggest that PDIA4 could be a novel prognostic marker for glioma.

\section{Data Sharing Statement}

The datasets used or analyzed during the current study are available from the corresponding author $(\mathrm{QM})$ on reasonable request. 


\section{Consent for Publication}

Written informed consent for publication was obtained from all participants.

\section{Ethics Approval and Consent to Participate}

About human tissues, the experimental use of surgical samples in this manuscript have been performed in accordance with the principles stated in the Declaration of Helsinki, also are approved by patients and Xiangya Hospital Ethics Committee. All patients were provided informed consent, and signed up voluntarily.

\section{Acknowledgments}

We gratefully thank China Scholarship Council for supporting us.

\section{Author Contributions}

All authors made substantial contributions to conception and design, acquisition of data, or analysis and interpretation of data; took part in drafting the article or revising it critically for important intellectual content; agreed to submit to the current journal; gave final approval of the version to be published; and agree to be accountable for all aspects of the work.

\section{Funding}

The study was supported by "National Natural Science Foundation of China" (Grant Number: 81872051) and the "Peking University Clinical Scientist Program" (Grant Number: BMU2019LCKXJ007).

\section{Disclosure}

The authors declare that they do not have any conflicts of interest for this work.

\section{References}

1. Ferreira WA, Pinheiro Ddo R, Costa Junior CA, et al. An update on the epigenetics of glioblastomas. Epigenomics. 2016;8(9):1289-1305. doi:10.2217/epi-2016-0040

2. Louis DN, Perry A, Reifenberger G, et al. The world health organization classification of tumors of the central nervous system: a summary. Acta Neuropathol. 2016;131(6):803-820. doi:10.1007/s00401-0161545-1

3. Molinaro AM, Taylor JW, Wiencke JK, et al. Genetic and molecular epidemiology of adult diffuse glioma. Nat Rev Neurol. 2019;15 (7):405-417. doi:10.1038/s41582-019-0220-2

4. Barthel FP, Johnson KC, Varn FS, et al. Longitudinal molecular trajectories of diffuse glioma in adults. Nature. 2019;576 (7785):112-120. doi:10.1038/s41586-019-1775-1
5. Ma Q, Long W, Xing C, et al. Cancer Stem Cells and Immunosuppressive Microenvironment in Glioma. Front Immunol. 2018;9:2924. doi:10.3389/fimmu.2018.02924

6. He Y, Long W, Liu Q. Targeting super-enhancers as a therapeutic strategy for cancer treatment. Front Pharmacol. 2019;10:361. doi:10.3389/fphar.2019.00361.

7. Eckel-Passow JE, Lachance DH, Molinaro AM, et al. Glioma groups based on 1p/19q, IDH, and TERT promoter mutations in tumors. $N$ Engl J Med. 2015;372(26):2499-2508. doi:10.1056/ NEJMoa1407279

8. Ostrom QT, Gittleman H, Truitt G, et al. CBTRUS statistical report: primary brain and other central nervous system tumors diagnosed in the United States in 2011-2015. Neuro-Oncology. 2018;20(suppl_4): iv1-iv86. doi:10.1093/neuonc/noy131

9. Rice T, Lachance DH, Molinaro AM, et al. Understanding inherited genetic risk of adult glioma - a review. Neuro-Oncol Practice. 2016;3 (1):10-16. doi:10.1093/nop/npv026

10. Ferrari DM, Soling H-D. The protein disulphide-isomerase family: unravelling a string of folds. Biochem J. 1999;339(1):1-10. doi:10.1042/bj3390001

11. Galligan JJ, Petersen DR. The human protein disulfide isomerase gene family. Hum Genomics. 2012;(6):6. doi:10.1186/1479-7364-6-6

12. Kuo TF, Chen TY, Jiang ST, et al. Protein disulfide isomerase a4 acts as a novel regulator of cancer growth through the procaspase pathway. Oncogene. 2017;36(39):5484-5496. doi:10.1038/onc.2017.156

13. Farquhar R, Honey N, Murant SJ, et al. Protein disulfide isomerase is essential for viability in Saccharomyces cerevisiae. Gene. 1991;108 (1):81-89. doi:10.1016/0378-1119(91)90490-3

14. Garbi N, Tanaka S, Momburg F, et al. Impaired assembly of the major histocompatibility complex class I peptide-loading complex in mice deficient in the oxidoreductase ERp57. Nat Immunol. 2006;7 (1):93-102. doi:10.1038/ni1288

15. Manukyan D, von Bruehl ML, Massberg S, et al. Protein disulfide isomerase as a trigger for tissue factor-dependent fibrin generation. Thromb Res. 2008;122(Suppl 1):S19-22. doi:10.1016/S00493848(08)70013-6

16. Ou W, Silver J. Role of protein disulfide isomerase and other thiol-reactive proteins in HIV-1 envelope protein-mediated fusion. Virology. 2006;350(2):406-417. doi:10.1016/j.virol.2006.01.041

17. Wang Z, Zhang H, Cheng Q. PDIA4: the basic characteristics, functions and its potential connection with cancer. Biomed Pharmacotherapy. 2019;122:109688. doi:10.1016/j.biopha.20 19.109688

18. Tufo G, Jones AW, Wang Z, et al. The protein disulfide isomerases PDIA4 and PDIA6 mediate resistance to cisplatin-induced cell death in lung adenocarcinoma. Cell Death Differ. 2014;21(5):685-695. doi:10.1038/cdd.2013.193

19. Samanta S, Tamura S, Dubeau L, et al. Expression of protein disulfide isomerase family members correlates with tumor progression and patient survival in ovarian cancer. Oncotarget. 2017;8 (61):103543-103556. doi:10.18632/oncotarget.21569

20. Yin F, Yi S, Wei L, et al. Microarray-based identification of genes associated with prognosis and drug resistance in ovarian cancer. J Cell Biochem. 2019;120(4):6057-6070. doi:10.1002/jcb.27892

21. Zhu Y, Xu H, Chen H, et al. Proteomic analysis of solid pseudopapillary tumor of the pancreas reveals dysfunction of the endoplasmic reticulum protein processing pathway. Molecular Cellular Proteomics. 2014;13(10):2593-2603. doi:10.1074/mcp.M114.038786

22. Xie J, Zhu Y, Chen H, et al. The immunohistochemical evaluation of solid pseudopapillary tumors of the pancreas and pancreatic neuroendocrine tumors reveals ERO1Lbeta as a new biomarker. Medicine. 2016;95(2):e2509. doi:10.1097/MD.0000000000002509

23. Zhao Z, Liu H, Wang $\mathrm{X}$, et al. Separation and identification of HSP-associated protein complexes from pancreatic cancer cell lines using 2D CN/SDS-PAGE coupled with mass spectrometry. $J$ Biomed Biotechnol. 2011;2011:193052. doi:10.1155/2011/193052 
24. Pawar H, Kashyap MK, Sahasrabuddhe NA, et al. Quantitative tissue proteomics of esophageal squamous cell carcinoma for novel biomarker discovery. Cancer Biol Ther. 2011;12(6):510-522. doi:10.4161/ cbt.12.6.16833

25. Chen N, Sun W, Deng X, et al. Quantitative proteome analysis of HCC cell lines with different metastatic potentials by SILAC. Proteomics. 2008;8(23-24):5108-5118. doi:10.1002/ pmic. 200800280

26. Su J, Long W, Ma Q, et al. Identification of a tumor microenvironment-related eight-gene signature for predicting prognosis in lower-grade gliomas. Front Genet. 2019. doi:10.3389/ fgene. 2019.01143

27. Tang Z, Li C, Kang B, et al. GEPIA: a web server for cancer and normal gene expression profiling and interactive analyses. Nucleic Acids Res. 2017;45(W1):W98-W102. doi:10.1093/nar/gkx247

28. Yu G, Wang LG, Han Y, et al. clusterProfiler: an R package for comparing biological themes among gene clusters. Omics. 2012;16 (5):284-287. doi:10.1089/omi.2011.0118

29. Su J, Ma Q, Long W, et al. LCTL is a prognostic biomarker and correlates with stromal and immune infiltration in gliomas. Front Oncol. 2019;9:1083. doi:10.3389/fonc.2019.01083

30. Yoshihara K, Shahmoradgoli M, Martinez E, et al. Inferring tumour purity and stromal and immune cell admixture from expression data. Nat Commun. 2013;4:2612. doi:10.1038/ncomms3612

31. Aran D, Hu Z, Butte AJ. xCell: digitally portraying the tissue cellular heterogeneity landscape. Genome Biol. 2017;18(1):220. doi:10.1186/ s13059-017-1349-1

32. Szklarczyk D, Gable AL, Lyon D, et al. STRING v11: protein-protein association networks with increased coverage, supporting functional discovery in genome-wide experimental datasets. Nucleic Acids Res. 2019;47(D1):D607-D613. doi:10.1093/nar/gky1131
33. Popescu NI, Lupu C, Lupu F. Extracellular protein disulfide isomerase regulates coagulation on endothelial cells through modulation of phosphatidylserine exposure. Blood. 2010;116(6):993-1001. doi:10.1182/blood-2009-10-249607

34. Schulman S, Bendapudi P, Sharda A, et al. Extracellular thiol isomerases and their role in thrombus formation. Antioxid Redox Signal. 2016;24(1):1-15. doi:10.1089/ars.2015.6530

35. Stopa JD, Zwicker JI. The intersection of protein disulfide isomerase and cancer associated thrombosis. Thromb Res. 2018;164(Suppl 1): S130-S135. doi:10.1016/j.thromres.2018.01.005

36. Reinhardt C, von Bruhl ML, Manukyan D, et al. Protein disulfide isomerase acts as an injury response signal that enhances fibrin generation via tissue factor activation. J Clin Invest. 2008;118 (3):1110-1122. doi:10.1172/JCI32376

37. Duan XF, Xin YW. Overexpression of molecule GRP94 favors tumor progression in lung adenocarcinoma by interaction with regulatory $\mathrm{T}$ cells. Thoracic Cancer. 2020;11:704-712. doi:10.1111/17597714.13321

38. Zhang Z, He T, Huang L, et al. Two precision medicine predictive tools for six malignant solid tumors: from gene-based research to clinical application. J Transl Med. 2019;17(1):405. doi:10.1186/ s12967-019-02151-8

39. Boussadia Z, Lamberti J, Mattei F, et al. Acidic microenvironment plays a key role in human melanoma progression through a sustained exosome mediated transfer of clinically relevant metastatic molecules. J Exp Clin Cancer Res. 2018;37(1):245. doi:10.1186/ s13046-018-0915-z

40. Han F, Xu Q, Zhao J, et al. ERO1L promotes pancreatic cancer cell progression through activating the Wnt/catenin pathway. J Cell Biochem. 2018;119(11):8996-9005. doi:10.1002/jcb.27155

\section{Publish your work in this journal}

OncoTargets and Therapy is an international, peer-reviewed, open access journal focusing on the pathological basis of all cancers, potential targets for therapy and treatment protocols employed to improve the management of cancer patients. The journal also focuses on the impact of management programs and new therapeutic agents and protocols on patient perspectives such as quality of life, adherence and satisfaction. The manuscript management system is completely online and includes a very quick and fair peer-review system, which is all easy to use. Visit http://www.dovepress.com/ testimonials.php to read real quotes from published authors. 